\title{
THE GLOBAL INVERSION PROBLEM: A CONFLUENCE OF MANY MATHEMATICAL TOPICS
}

\author{
F. Xavier
}

Dedicated to Professor Manfredo do Carmo on the occasion of his $80^{\text {th }}$ birthday

\begin{abstract}
The question of deciding when a locally invertible map admits a global inverse is one of obvious importance, with applications and connections to many different areas of mathematics. In this paper we survey some of the recently discovered invertibility mechanisms, rooted in global analysis, algebraic and differential geometry, topology, complex analysis and dynamical systems.
\end{abstract}

\section{Introduction}

Various existence and uniqueness problems arising in algebraic geometry, complex analysis, nonlinear analysis and dynamical systems, as well as various branches of applied mathematics, can be subsumed under a single, unifying theme:

Programme. Let $M$ be an $n$-dimensional non-compact differentiable manifold, and $f: M \rightarrow \mathbb{R}^{n}$ a smooth local diffeomorphism. Identify the general topological, analytic and geometric mechanisms that force the map $f$ to be injective.

The aim of this paper is to report on some of the work done in recent years by the present author and his collaborators on various aspects of the above Programme. To be sure, the issue of global invertibility has been examined by many other authors in the past, but mainly from the analytic and algebraic standpoints. For a sampler, we refer the reader to [B1], [B2], [BW], [CMe], $[\mathrm{D}],[\mathrm{E}],[\mathrm{Fe}],[\mathrm{H}],[\mathrm{G}],[\mathrm{GLS}],[\mathrm{Gu}],[\mathrm{M}],[\mathrm{O}],[\mathrm{P}],[\mathrm{R}],[\mathrm{So}],[\mathrm{W}],[\mathrm{Y}],[\mathrm{Z}]$, and the references therein. Here, we shall concentrate on other aspects of the problem, most notably the ones that have a topological or geometric flavor. 
Since this (admittedly incomplete) survey is meant to appear on a Volume commemorating the many scientific accomplishments of Manfredo do Carmo the founder of the Brazilian School of Differential Geometry -, special emphasis will be placed on interactions with differential geometry itself but, to the extent possible, we will also discuss results originating in other areas of mathematics.

The organization of this paper is as follows. In Section 2 we provide several examples of central topics where the issue of global invertibility comes up naturally, explaining along the way how the topics themselves are interconnected. In the remaining sections we elaborate on some fairly recent topological, analytic, dynamic and geometric results that complement our discussion in Section 2.

The presentation is interspersed with several open problems, no doubt an attempt to lure the unsuspecting mathematician to this beautiful corner of our discipline.

\section{A cornucopia of topics}

We begin with a brief discussion of some important topics related to the above Programme. Although at first they look disparate, as we shall see in the course of our discussion they are actually connected at several different levels.

Topic I: The classical theory of univalent functions. A conformal orientation-preserving local diffeomorphism that is defined in the open unit $\operatorname{disc} D=\{z \in \mathbb{C}:|z|<1\}$ and takes values into $\mathbb{R}^{2}$ can be viewed as a holomorphic function $f: D \rightarrow \mathbb{C}$. Of special interest is the case when $f$ is univalent, that is, injective. The class $S$ of all holomorphic univalent functions in $D$ satisfying $f(0)=0$ and $f^{\prime}(0)=1$ was the object of much study in the last century, culminating with the solution by de Branges [Br], [S] of the celebrated Bieberbach conjecture: for any $f \in S$, the estimate $\left|f^{(k)}(0)\right| \leq k k$ ! holds for all $k \geq 2$. Equivalently, $\left|f^{(k)}(0)\right| \leq k k !\left|f^{\prime}(0)\right|$ for any injective holomorphic 
function on $D$.

The case $k=2$, due to Bieberbach, yields the so-called distortion theorems which, in turn, imply the compactness of the class $S$ ([S], p. 264). Thus, the basic estimate $\left|f^{\prime \prime}(0)\right| \leq 4$ for $f \in S$, most commonly written in the form $\left|a_{2}\right| \leq 2$ where $f(z)=z+a_{2} z^{2}+\cdots$, already yields important qualitative information. In particular, it follows from the compactness of $S$ that there are constants $C_{k}$ such that $\left|f^{(k)}(0)\right| \leq C_{k}\left|f^{\prime}(0)\right|$ for every $k \geq 2$ and injective holomorphic function $f$ on $D$. The Bieberbach conjecture (the de Branges theorem) asserts that one can take $C_{k}$ to be $k k$ !.

The Bieberbach estimate $\left|f^{\prime \prime}(0)\right| \leq 4\left|f^{\prime}(0)\right|$ has recently been generalized by F. Fontenele and the author $[\mathrm{FX}]$ to the case of arbitrary conformal injective $f$ : $D \rightarrow \mathbb{R}^{n}, n \geq 3$. The new estimate involves two correction terms. The first one is geometric, coming from the second fundamental form of the image surface. The second term is of a dynamical nature and involves certain Riemannian quantities associated to conformal attractors. As we shall see in Section 4, this generalized Bieberbach estimate leads to a natural conjecture in the theory of embedded minimal surfaces.

Topic II: The asymptotic stability conjecture. Consider a smooth vector field $X$ on the plane, vanishing at zero. If the eigenvalues of $D X(0)$ have negative real parts, an elementary result shows that every trajectory of $X$ that starts near 0 will be attracted to the origin. The asymptotic stability conjecture, also known as the Markus-Yamabe conjecture, claimed that if the eigenvalues of $D X(z)$ have negative real part, for all $z \in \mathbb{R}^{2}$, then 0 is a global attractor in the sense that every positive trajectory of $X$ converges to the origin.

By a theorem of Olech $[\mathrm{O}]$, it was known that the conjecture would follow if it could be established that $X$, when viewed as a map from $\mathbb{R}^{2}$ into itself, is injective. This was accomplished by Gutierrez [Gu] (see also Glutsiuk $[\mathrm{G}]$ and 
Fessler $[\mathrm{Fe}])$. The solution by Gutierrez is specially interesting to us. In fact, he proved the following stronger result: If a local diffeomorphism $F: \mathbb{R}^{2} \rightarrow \mathbb{R}^{2}$ is such that $[0, \infty) \cap \operatorname{Spec} D F(z)=\emptyset$ for every $z \in \mathbb{R}^{2}$, then $F$ is injective. In Section 5 we propose a considerable strengthening of the Gutierrez theorem, casting the result in a differential-geometric context.

Topic III: The jacobian conjecture. This well-known problem in algebraic geometry claims that if $\mathbb{K}$ is an algebraically closed field and $F: \mathbb{K}^{n} \rightarrow \mathbb{K}^{n}$, $n \geq 2$, is a polynomial map with constant jacobian determinant, then $F$ must be bijective $[\mathrm{BW}],[\mathrm{E}]$. It is known that it suffices to consider the case $\mathbb{K}=\mathbb{C}$, and to show that $F$ is injective. Notice that for polynomial maps $F: \mathbb{C}^{n} \rightarrow \mathbb{C}^{n}$, the jacobian determinant is constant if and only if $F$ is a holomorphic immersion. In short, the jacobian conjecture can be reformulated as follows: Every polynomial local biholomorphism $F: \mathbb{C}^{n} \rightarrow \mathbb{C}^{n}$ is injective.

Despite considerable work, which led to the solution of the conjecture in some special cases, in its full generality the problem remains open, even in case of two variables. In $[\mathrm{NTX}]$, topological arguments are used to prove that the jacobian conjecture is true in a "generic" sense (see Section 4 for details).

The search for a solution to the jacobian conjecture can be seen as being part of the much larger (and seemingly harder) task of understanding the structure of the group of biholomorphisms of $\mathbb{C}^{n}$, when $n \geq 2$. This is the subject of the next topic.

Topic IV: Automorphisms of $\mathbb{C}^{n}$. The group Aut $\left(\mathbb{C}^{n}\right)$ of biholomorphisms of $\mathbb{C}^{n}$ is largely unknown if $n>1$. In stark contrast, Aut $(\mathbb{C})$ is rather small, consisting of the non-constant affine linear maps. The description of Aut $(\mathbb{C})$ follows from the observation that an injective holomorphic function $f: \mathbb{C} \rightarrow \mathbb{C}$ satisfying $f(0)=0$ and $f^{\prime}(0)=1$ must be the identity. These considerations suggest that similar characterizations of the identity might be useful in understanding the structure of Aut $\left(\mathbb{C}^{n}\right)$. 
On the other hand, any expectations in this regard should be tempered by the fact that injective entire maps are not necessarily in Aut $\left(\mathbb{C}^{n}\right)$ if $n \geq 2$, as shown by the classical examples given by Fatou and Bieberbach ([BM], p. 45), where the image of the map omits a non-empty open set. Another consideration is the fact that the identity cannot be characterized, if $n \geq 2$, solely by finitely many pointwise conditions at zero. In order to see this, one can take the automorphisms of $\mathbb{C}^{2}$ given by $F(x, y)=(x+p(y), y)$, where the polynomial $p$ is arbitrary. In particular, $F$ can be made to coincide with the identity up to an arbitrarily high order simply by taking $p(y)=y^{d}$ with $d$ sufficiently high.

The above discussion shows that some kind of global condition is necessary if one is to prove a rigidity theorem for the identity $I_{\mathbb{C}^{n}}$ in higher dimensions. Such a condition was found in [X3] and will be discussed in Section 4.

\section{Global Inversion: Topological and real-analytic arguments}

A basic result in Riemannian geometry, the Cartan-Hadamard theorem, states that the exponential map at any point of a complete simply-connected manifold of non-positive sectional curvature is a diffeomorphism. From a geometric standpoint, what makes the theorem true is the fact that the exponential map does not decrease distance or, what is the same, that the inverse of the differential of the exponential map has norm bounded by one.

The same kind of argument applies to local diffeomorphisms of $\mathbb{R}^{n}$, yielding what is known as the Hadamard Theorem in the classical literature on the theory of ordinary differential equations. In fact, the topological arguments involved are fairly basic facts about covering spaces and proper maps (but not local compactness), and so they work for infinite dimensional spaces as well:

Theorem 1 ([P]). Let $X$ be a Banach space and $f: X \rightarrow X$ a smooth local 
diffeomorphism. If

$$
\sup _{x \in X}\left\|D f(x)^{-1}\right\|<\infty
$$

then $f$ is bijective.

In [NX1] the authors used degree theory to show that the above theorem can be substantially improved if $\operatorname{dim} X<\infty$ :

Theorem 2 ([NX1]). Let $f: \mathbb{R}^{n} \rightarrow \mathbb{R}^{n}$ be smooth. For $v \in \mathbb{R}^{n}$, let $S_{v}=$ $\left\{D f(x)^{*} v: x \in \mathbb{R}^{n}\right\}$.

(a) If $0 \notin S_{v}$ for each non-zero $v \in \mathbb{R}^{n}$, then $f$ is locally invertible.

(b) If $0 \notin \overline{S_{v}}$ for each non-zero $v \in \mathbb{R}^{n}$, then $f$ is globally invertible.

Observe that condition (b) amounts to

$$
\inf _{x \in \mathbb{R}^{n}}\left\|D f(x)^{*} v\right\|=\inf _{x \in \mathbb{R}^{n}}\|\nabla\langle f, v\rangle(x)\|>0, \quad v \neq 0 .
$$

Part (a) is the usual inverse function theorem and is included in the statement of the theorem for comparison purposes only. Thus, the passage from local to global injectivity is achieved by stipulating that 0 should not be in the closure of $S_{v}$, for every non-zero $v$. Consider the containment

$$
\bigcup_{v \neq 0} \overline{S_{v}} \subset \overline{\bigcup_{v \neq 0} S_{v}}
$$

Condition (b) asks only that

$$
0 \notin \bigcup_{v \neq 0} \overline{S_{v}}
$$

The stronger condition

$$
0 \notin \overline{\bigcup_{v \neq 0} S_{v}}
$$

turns out to be simply a rewriting of the Hadamard condition in Theorem 1 above. This subtle distinction is illustrated by the simple map $f: \mathbb{R}^{2} \rightarrow \mathbb{R}^{2}$, 
given by $f(x, y)=\left(x+y^{3}, y\right)$. The map $f$ is clearly injective, a fact that is picked up by Theorem 2 but not by Theorem 1 (see [NX1], p. 19).

The basic idea in the proof of Theorem 2 is to create a mechanism that works as a "non-linear adjoint", transforming injectivity questions into problems about surjectivity that can then be handled using degree theory.

Example 1. Every quadratic polynomial local diffeomorphism of $\mathbb{R}^{n}$ into itself is bijective. Indeed, for a given $v \neq 0$ the set $S_{v}$ is an affine hyperplane, hence closed. Since $f$ is a local diffeomorphism, $0 \notin S_{v}=\overline{S_{v}}$, and the result follows from the above theorem. The first proof of the invertibility of quadratic polynomial with constant jacobian determinant was given by Wang [W], using algebraic arguments. Thanks to the work of Yagzhev [Y], Druzkowski [D], and Bass-Connell-Wright $[\mathrm{BW}]$, it is known that it suffices to settle the jacobian conjecture for certain types of cubic polynomial maps.

As it was mentioned before, the proof of Theorem 2 is based on arguments involving degree theory, and so they cannot be directly extended to the infinite dimensional setting. On the other hand, various degree theories have been introduced in infinite dimensions, for suitably restricted classes of maps. The main argument in [NX1] extends verbatim to the infinite dimensional situation, but it is not clear what kind of differential condition on $f$ should be imposed in order for the relevant maps to be admissible for degree theory. A positive solution to the problem below would quite likely have interesting applications in non-linear analysis.

Problem 1. Under what additional structural conditions on the differential of a smooth Banach space local diffeomorphisms $f: X \rightarrow X$ is the condition

$$
\inf _{x \in X}\left\|D f(x)^{*} v\right\|>0, v \neq 0,
$$

sufficient to guarantee that $f$ is bijective? What if $D f(x)$ is a suitable Fredholm 
operator, perhaps a compact perturbation of the identity?

Another insight into the global injectivity question, one that has been proven rather fruitful, is the following nearly tautological observation: a local diffeomorphism $f: \mathbb{R}^{n} \rightarrow \mathbb{R}^{n}$ is injective if and only if the pre-image of every point is a connected set. Can one infer injectivity from the knowledge that the pre-images of certain positive dimensional submanifolds are connected?

In regards to this question, it can be shown that (b) in Theorem 2 implies directly that the pre-image of every affine hyperplane is connected. Earlier, S. Nollet and the author had noticed that any map satisfying the hypotheses of the jacobian conjecture (Topic III) automatically possesses the property that the pre-image of every real hyperplane in $\mathbb{R}^{2 n} \cong \mathbb{C}^{n}$ is connected. This is a consequence of a classical theorem of Bertini in algebraic geometry (apart from some exceptional situations, the generic element in a pencil is irreducible). We were then led to ask

Problem 2. Let $f: \mathbb{R}^{n} \rightarrow \mathbb{R}^{n}$ be a local diffeomorphism satisfying the property that the pre-image of every affine hyperplane is non-empty and connected. Must $f$ be bijective?

In his 2006 University of Notre Dame Dissertation (see also [B1]), E. Cabral Balreira gave an affirmative solution to this problem under a stronger assumption on the topology of the pre-images of the affine hyperplanes. Recall that a topological space is called acyclic if it has the homology of a point.

Theorem 3 ([B1]). Let $f: \mathbb{R}^{n} \rightarrow \mathbb{R}^{n}$ be a local diffeomorphism.

(a) If the pre-image of every affine hyperplane is empty or acyclic, then $f$ is injective.

(b) If the pre-image of every affine hyperplane is non-empty and acyclic, then $f$ is bijective. 
Observe that the second part of Theorem 3 provides a condition that is both necessary and sufficient for a local diffeomorphism to be bijective. As remarked before, Theorem 1 follows from Theorem 2. Using simple arguments from Morse theory one can see that Theorem 2 is a consequence of Theorem 3.

Recent discoveries have added considerably to our understanding of the phenomenon of global injectivity. This is exemplified by the three theorems discussed in this section, based on increasingly more sophisticated topological arguments: covering spaces theory, degree theory, and intersection theory.

\section{Global Inversion: Topological and complex- analytic arguments}

We now turn our attention to the jacobian conjecture (Topic III). Let then $F: \mathbb{C}^{n} \rightarrow \mathbb{C}^{n}$ be a polynomial local biholomorphism. Recall that (JC) claims that $F$ must be injective. From general principles in algebraic geometry, it is known that there exists an algebraic complex hypersurface $D \subset \mathbb{C}^{n}$ (possibly reducible and with singularities) such that the induced map $\mathbb{C}^{n}-F^{-1}(D) \rightarrow$ $\mathbb{C}^{n}-D$ is a $d$-sheeted covering map where $d<\infty$. Injectivity will follow if one can show $d=1$.

We summarize below the study made in $[\mathrm{NTX}]$ of the topological properties of $D$ in a possible counterexample to the jacobian conjecture.

An algebraic hypersurface $D \subset \mathbb{C}^{n}$ is given by a polynomial equation $f\left(z_{1}, \cdots, z_{n}\right)=0$. A theorem of Verdier $[\mathrm{V}]$ states that the corresponding map $f: \mathbb{C}^{n} \rightarrow \mathbb{C}$ is a locally trivial topological fibration away from a finite set subset of $\mathbb{C}$. The smallest such set is called the bifurcation locus of $f$, and is denoted by $B_{f}$. The bifurcation locus contains the images of critical points of $f$, but it may also contain other points, coming from "singularities at infinity". The hypersurface $D$ will be called non-bifurcated if $0 \notin B_{f}$, where $f$ is a polynomial of minimal degree defining $D$. 
Theorem $4([\mathbf{N T X}])$. Fix $n>1$ and let $D \subset \mathbb{C}^{n}$ be a smooth connected nonbifurcated hypersurface. If $F: X \rightarrow \mathbb{C}^{n}$ is a local diffeomorphism of simply connected manifolds which is a d-fold covering map away from $D$, then $d=1$ or $d=\infty$.

The proof of the above dichotomy is based on: i) a counting argument that uses the orientability of complex hypersurfaces in a crucial way, ii) separation properties of real hypersurfaces, and iii) arguments from surgery theory.

After certain geometric constructions, the relevant question becomes the following: Given an algebraic hypersurface $D$ of $\mathbb{C}^{n}$, is there a real hypersurface $V$ such that $\partial V=D$ and $\mathbb{C}^{n}-V$ is simply-connected? When $n=1$ an algebraic hypersurface is simply a finite number of points in $\mathbb{C}$, and one can take $V$ to be the union of non-intersecting infinite rays emanating from the points of $D$. Realizing the condition $\partial V=D$ is easy in any dimension, but there are obstructions if $n \geq 2$ to make the complement of $V$ simply-connected. It is precisely to achieve this that surgery theory is used, in order to kill certain homotopy classes.

If the map $F$ in Theorem 4 is algebraic the alternative $d=\infty$ cannot occur, and thus one obtains the following application to the jacobian conjecture.

Theorem $5([\mathbf{N T X}])$. Let $D \subset \mathbb{C}^{n}$ be a smooth connected non-bifurcated hypersurface. If $F: \mathbb{C}^{n} \rightarrow \mathbb{C}^{n}$ is a polynomial map with non-vanishing jacobian determinant and $\# F^{-1}(q)=\operatorname{deg} F$ for $q \notin D$, then $F \in A u t\left(\mathbb{C}^{n}\right)$.

Next, we consider the global invertibility problem in the context of general local biholomorphisms, not necessarily algebraic. But first we discuss an example where differential geometry plays a key role.

Example 2. Let $F: \mathbb{C}^{n} \rightarrow \mathbb{C}^{n}, n \geq 2$, be a local biholomorphism with the property that the pre-image of every complex line is both connected and simply connected. We claim that $F$ is injective. 
Indeed, if $F$ is not injective we may suppose that $F(p)=F(q)=0$, with $p \neq q$, so that $F^{-1}(l)$ contains both $p$ and $q$ for all one-dimensional complex subspaces $l$. It is easy to see that the complex curve $F^{-1}(l)$ (= an open Riemann surface) is properly embedded in $\mathbb{C}^{n}$, whether $F$ is a proper map or not. Hence, with respect to the induced flat Riemannian metric of $\mathbb{C}^{n}, F^{-1}(l)$ is a complete simply connected real surface.

Since $F^{-1}(l)$ is a complex submanifold of $\mathbb{C}^{n}$, it has non-positive curvature. Hence, $F^{-1}(l)$ is a Hadamard surface, so that any two points can be joined by a unique geodesic. Given $l \in \mathbb{P}^{n-1}$, let $w(l)$ denote the initial vector of the (unique) unit-speed geodesic along $F^{-1}(l)$ joining $p$ to $q$, and set $v(l)=$ $d F(p) w(l) \in T_{l, 0}$.

Notice that, as $l$ varies inside $\mathbb{C P}^{n-1}$, all geodesic segments are contained in a fixed compact set of $\mathbb{C}^{n}$. The map $v$ is continuous because geodesics converge to geodesics in the $C^{2}$ topology (which, after passing to subsequences, is a consequence of $C^{3}$ uniform boundedness) and from the uniqueness of the geodesic along $F^{-1}(l)$. Since $v$ is non-vanishing, it is clear that the map $\mathbb{C P}^{n-1} \rightarrow S^{2 n-1}$, given by $l \rightarrow v(l) /|v(l)|$, is a continuous section of the Hopf map $\pi: S^{2 n-1} \rightarrow \mathbb{C P}^{n-1}$ (the latter is the natural map that associates to a point in the unit sphere the unique complex line joining the origin to the point in question). But this is a contradiction since, as it is well known, the Hopf map admits no continuous sections. For instance, the composite map in cohomology

$$
H^{2}\left(\mathbb{C P}^{n-1}\right) \rightarrow H^{2}\left(S^{2 n-1}\right) \rightarrow H^{2}\left(\mathbb{C P}^{n-1}\right),
$$

induced by a continuous section, would be the identity. But this is impossible, since $H^{2}\left(\mathbb{C P}^{n-1}\right) \neq 0$ whereas $H^{2}\left(\mathbb{S}^{2 n-1}\right)=0$.

For some questions in complex analysis curvature considerations are indispensable. For instance, the invariant form of the Schwarz lemma simply says that every holomorphic self-map of the open unit disc in $\mathbb{C}$ is distance 
non-increasing, relative to the Poincaré metric. The question then arises as to whether the role of curvature in the above example is essential, or merely a technical artifact. In [NX2], the authors prove a theorem, using similar arguments, that is curvature-free.

To explain the result, we start by defining a rigid domain $D \subset \mathbb{C P}^{1}$ to be an open domain that can be holomorphically embedded in $\mathbb{C P}^{1}$ in a unique way, up to Moebius transformations. For instance, the complement of a finite set in $\mathbb{C}$ (a connected rational curve, in the algebraic geometry terminology) is rigid. By contrast, any simply-connected subset of $\mathbb{C}$, other than $\mathbb{C}$ itself, is highly non-rigid by the Riemann mapping theorem.

Theorem 6 ([NX2]). Let $X$ be a connected complex manifold of dimension $n \geq 2, F: X \rightarrow \mathbb{C}^{n}$ a local biholomorphism. Fix $q \in F(X)$ and suppose that $F^{-1}(l)$ is conformal to a rigid domain $D_{l}$ for every complex line $l$ passing through $q$. Then $q$ is assumed exactly once by $F$.

Next, we turn our attention to Topic IV in Section 2. As we indicated there, in $[\mathrm{X} 3]$ we were able to prove a rigidity theorem for the identity in $\mathbb{C}^{n}$, among univalent local biholomorphisms:

Theorem 7 ([X3]). An injective local biholomorphism $f: \mathbb{C}^{n} \rightarrow \mathbb{C}^{n}$ is the identity if and only if

(i) The power series at 0 of $f-I$ has no terms of order $\leq 2 n+1$.

(ii) The function $|\operatorname{det} D f(z)||z|^{2 n}|f(z)|^{-2 n}$ is subharmonic on $\mathbb{C}^{n}$.

Problem 3. One would like to improve the vanishing condition in (i) to $\leq 2 n-1$, or even replace it with $f(0)=0, D f(0)=I$. If this could be done the resulting statement would be sharp, regarding the dimension, already when $n=1$. Indeed, when $n=1$ the function in (ii) can be realized as the abso- 
lute value squared of a holomorphic function, and therefore it is automatically subharmonic.

The arguments in [X3] are essentially of a real-variables nature, although they are inspired by the proof of the classical Bieberbach estimate (see Topic I). In the classical setting, an important role is played by the complex inversion $z \rightarrow z^{-1}$. The main idea in the higher dimensional case is to use the inversion in the unit sphere, to the extent that is possible, as a substitute for the complex inversion. At the conceptual level, Fourier (power) series are then replaced by spherical harmonics.

We close this section by making some comments about the embeddednes question for minimal surfaces in $\mathbb{R}^{3}$. Although we are no longer working in the realm of local diffeomorphisms, embeddednes of surfaces can clearly be cast as an injectivity question. A simply-connected immersed minimal surface $M$ in $\mathbb{R}^{3}$ is obtained as the image of a conformal harmonic immersion $f: \Omega \rightarrow$ $\mathbb{R}^{3}$, where $\Omega$ is either the open unit disc $D \subset \mathbb{C}$, or $\mathbb{C}$ itself. The only such examples $M$ that are proper and embedded (i.e., $f$ is injective) are planes and helicoids. This important theorem was established by Meeks-Rosenberg [MR] using, among other arguments, results from the Colding-Minicozzi theory of embedded minimal discs (see, e.g. [CM1]-[CM4]).

The classical link between the theory of minimal surfaces and complex analysis has been explored, with great success, to tackle other fundamental geometric problems. Given the history of the subject, one would like to have a complex-analytic interpretation of the works of Colding-Minicozzi and MeeksRosenberg, with the hope that more could be revealed about the structure of embedded minimal discs.

In this context, a central theme is the role of the conformal type in the embeddedness question for minimal surfaces. In particular, one would like to 
know, in the Meeks-Rosenberg theorem, if parabolicity alone suffices:

Problem 4. If $g: \mathbb{C} \rightarrow \mathbb{R}^{3}$ is a non-flat conformal harmonic embedding, must $g(\mathbb{C})$ be a helicoid?

There is a vague and yet compelling analogy between the theory of conformal harmonic embeddings of the open unit disc $D$ into $\mathbb{R}^{3}$, and the theory of holomorphic univalent functions on $D$ (Topic I). Below is one aspect of this relationship.

It is an easy matter to use the Bieberbach estimate $\left|f^{\prime \prime}(0)\right| \leq 4\left|f^{\prime}(0)\right|$ and a scaling argument to retrive the well known fact that the only univalent entire functions are of the form $f(z)=a z+b, a \neq 0$. One ought to regard the last statement as the complex analytic form of the (much harder) Problem 4. Indeed, in both cases one would have scarcity of injective objects.

As mentioned in our discussion of Topic I in Section 2, the Bieberbach estimate $\left|f^{\prime \prime}(0)\right| \leq 4\left|f^{\prime}(0)\right|$ has been generalized recently by F. Fontenele and the author $[\mathrm{FX}]$ to the case of arbitrary conformal embeddings $f: D \rightarrow \mathbb{R}^{n}$. At present, however, it is not clear how these results can be used to study Problem 4.

\section{Global Inversion: Geometric and dynamical arguments (I)}

We begin by elaborating on our comments about Topic III, Section 2. The global stability conjecture (also known as the Markus -Yamabe conjecture), stated that if $X$ is a sufficiently smooth vector field in the plane such that $X(0)=0$ and the real parts of the eigenvalues of the matrices $D X(z)$ have negative real part, for all $z \in \mathbb{R}^{2}$, then the origin is a global attractor. In other words, every forward trajectory $\phi_{t}(z)$ of $X$ is defined for all positive times and satisfies $\lim _{t \rightarrow \infty} \phi_{t}(z)=0$. 
We recall the famous Gutierrez Injectivity Theorem $[\mathrm{Gu}]$, which implies the Markus-Yamabe conjecture: If a local diffeomorphism $F: \mathbb{R}^{2} \rightarrow \mathbb{R}^{2}$ is such that $[0, \infty) \cap \operatorname{Spec} D F(z)=\emptyset$ for every $z \in \mathbb{R}^{2}$, then $F$ is injective. Before we go on to propose a geometric framework for the Gutierrez theorem, we provide some elementary examples to illustrate the truly global nature of his result.

It is natural to inquire if injectivity is a consequence of the spectral condition $[0, \infty) \cap$ Spec $D F(z)=\emptyset$, where the map $F$ is now defined only on a convex subset $\Omega$ of the plane. This question has a negative answer, as shown by the following simple example. Take $\Omega$ to be the open upper half plane in $\mathbb{C}$ and consider the real map underlying $F: \Omega \rightarrow \mathbb{C}, F(z)=z^{3}$. For $z \in \Omega$, the derivative $F^{\prime}(z)=3 z^{2}$ misses $[0, \infty)$ and yet $F$ is not one-to-one.

Despite the observation in the last paragraph, under a suitable spectral condition a local diffeomorphism defined on a convex set can be shown to be injective: If $\Omega \subset \mathbb{R}^{2}$ is open, convex and $F: \Omega \rightarrow \mathbb{C}$ satisfies the stronger spectral condition $\mathbb{R} \cap \operatorname{Spec} D F(z)=\emptyset$ for all $z \in \Omega$, then $F$ is injective.

To see this, suppose $F(a)=F(b)$, with $a \neq b$, and set $v=b-a$. The function $g$ on $[0,1]$ given by $g(t)=\left\langle F(a+t v), v^{\perp}\right\rangle$ satisfies $g(0)=g(1)$. By the mean value theorem, there is a point $z$ in the line segment $[a, b]$ such that $\left\langle D F(z) v, v^{\perp}\right\rangle=0$. In particular, $v$ is an eigenvector of $D F(z)$ corresponding to a real eigenvalue, a contradiction.

Differential geometry provides concrete examples of planar maps whose jacobians have no real eigenvalues. Let $M \subset \mathbb{R}^{3}$ be a surface of positive curvature that is given by the graph of a function $f$ defined on a convex subset $\Omega$ of $\mathbb{R}^{2}$. Consider now the map $F: \Omega \rightarrow \mathbb{R}^{2}$,

$$
F(x, y)=\left(\frac{\partial f}{\partial y},-\frac{\partial f}{\partial x}\right)=-J \nabla f,
$$

where $J$ is the complex structure. Since $D F$ is traceless and $\operatorname{det} D F>0$, the eigenvalues of $D F$ are purely imaginary. By the argument in the preceding paragragh, $F$ is injective. 
Notice that the last conclusion is equivalent to the fact that the Gauss map $\mathcal{G}: M \rightarrow S^{2}$,

$$
\mathcal{G}(x, y)=\left(1+|\nabla f|^{2}\right)^{-\frac{1}{2}}\left(-f_{x},-f_{y}, 1\right)
$$

of a graph $z=f(x, y)$ of positive curvature, is necessarily injective if $(x, y)$ runs over a convex domain (recall that the sign of the curvature is the sign of the quantity $f_{x x} f_{y y}-f_{x y}^{2}$ ). Here, the condition of convexity is essential. Indeed, there exist graphs of positive curvature (over non-convex domains, obviously) that have non-injective Gauss maps. One such explicit example was kindly communicated to us by M. Ghomi (who also draw our attention to [AG], [Gh1] and [Gh2]). In fact, as it will be shown below, it is not difficult to construct such examples.

Example 3. Start with the arc $\Gamma$ of the unit circle given by $\Gamma=\{(\cos \theta, \sin \theta) \mid-$ $\left.\frac{\pi}{2} \leq \theta \leq \pi\right\}$. Let $a=a(\theta), b=b(\theta)$ be positive smooth functions on $\left[-\frac{\pi}{2}, \pi\right]$ such that

$$
\int_{-\frac{\pi}{2}}^{\pi} a(\theta) \sin \theta=\int_{-\frac{\pi}{2}}^{\pi} b(\theta) \cos \theta=0 .
$$

One can see, either arguing directly or by invoking the Whitney extension theorem, that there is a smooth function $f$, defined in a neighborhood of $\Gamma$, such that, along $\Gamma$, one has $f_{x x}=a, f_{x y}=0, f_{y y}=b$. One can then compute, along $\Gamma$,

$$
\frac{d}{d \theta}\left(f_{x}\right)=-a \sin \theta, \frac{d}{d \theta}\left(f_{y}\right)=b \cos \theta
$$

The above orthogonality conditions imply that $\nabla f$ takes on the same value at $(0,-1)$ and $(-1,0)$. In particular, the graph $z=f(x, y)$, for $(x, y)$ in a sufficiently small (hence non-convex) neighborhood of $\Gamma$ has positive curvature $\left(f_{x x} f_{y y}-f_{x y}^{2}>0\right.$ near $\left.\Gamma\right)$ and non-injective Gauss map.

To sum up, we have seen that the Gutierrez theorem fails for convex subsets of the plane; also, under the stronger assumption that the spectra of the jacobians miss the real line, convexity of the domain implies injectivity. Besides, 
the last conclusion may fail if the domain in question is not convex. In [SX], Thm. 3, we proved a "hybrid "injectivity theorem, involving both spectral conditions:

(•) If the $C^{1}$ map $F: B(r) \rightarrow \mathbb{R}^{2}$ satisfies $[0, \infty) \cap \operatorname{Spec} D F(z)=\emptyset \forall z \in B(r)$ and $\mathbb{R} \cap \operatorname{Spec} D F(z)=\emptyset \forall z \notin B(r / \sqrt{2})$, then $F$ is injective on $B(r / \sqrt{2})$.

Problem 5. Is the constant $\sqrt{2}$ that appears in the above theorem the best possible?

In exploring the relationship between the phenomenon of global injectivity and the eigenvalues of the Jacobian matrix, one cannot fail to mention the Chamberland conjecture, which is more general than the jacobian conjecture:

Problem 6. (Chamberland) If $F: \mathbb{R}^{n} \rightarrow \mathbb{R}^{n}$ is a $C^{1}$ map and the eigenvalues of $D F(p)$ are globally bounded away from zero, then $F$ is injective.

Next, we return to our original theme of understanding the Gutierrez theorem from a geometric point of view. Consider two smooth oriented planar foliations. We say that they are loosely transversal if, for every point of their common domain, either the leaves are transversal or they are tangent but have opposite orientations at the tangency point.

It is not difficult to see that the spectral condition $[0, \infty) \cap \operatorname{Spec} D F(z)=\emptyset$ in the Gutierrez theorem is equivalent to the geometric condition that every foliation of $\mathbb{R}^{2}$ by parallel lines is loosely tranversal to its pull-back foliation under the local diffeomorphism. Likewise, the stronger spectral condition $\mathbb{R} \cap$ Spec $D F(z)=\emptyset$ is equivalent to the condition that the above foliations are actually transversal.

In the context of Hadamard surfaces (i.e., complete simply connected surfaces of non-positive curvature), parallel lines are horocycles corresponding to the same point at infinity. One is thus led to the following problem, meant to 
provide a geometrization of the Gutierrez Injectivity Theorem:

Problem 7. Let $f:(M, g) \rightarrow(M, g)$ be an orientation-preserving local diffeomorphism of an oriented Hadamard surface. Suppose that for every ideal point $v \in M(\infty)$, the horocycle foliation $H_{v}$ and its pull-back foliation $f^{*}\left(H_{v}\right)$ are loosely transversal. Is it true that $f$ must be injective?

If $(M, g)$ is the flat plane the answer is yes, as given by the Gutierrez theorem. In [X1] we used degree theory to answer this question in the affirmative, under the additional assumption that, asymptotically, the foliations $H_{v}$ and $f^{*}\left(H_{v}\right)$ are actually transversal, for all $v \in M(\infty)$.

The arguments of Gutierrez are based on the analysis of the so-called HalfReeb components. It is quite possible that his original arguments carry over to the geometric setting of Problem 7. Of course, one would still need a "dictionary"to go from the linear (flat) case to general Hadamard surfaces (e.g., linear functions correspond to Busemann functions).

One should also point out that the natural generalization of the theorem of Gutierrez does not hold if $n \geq 3$, although one can prove a global injectivity theorem with nearly spectral hypotheses ([SX]).

\section{Global Inversion: Geometric and dynamical arguments (II)}

We end this survey with a class of geometric theorems where the key insight comes from dynamics. Given a local diffeomorphism $f: \mathbb{R}^{n} \rightarrow \mathbb{R}^{n}$, the following naive idea comes to mind in trying to prove that $f$ is bijective. Let $q$ be a point in $\mathbb{R}^{n}$ and consider the $n$ affine coordinate hyperplanes through $q$, say $H_{1}, \cdots, H_{n}$. The point $q$ will be in the image of $f$ if and only if the hypersurfaces $f^{-1}\left(H_{j}\right), 1 \leq j \leq n$, have a non-empty intersection. Furthermore, $q$ is covered only once if the intersection of these hypersurfaces reduces to a single point. 
One is then led to the following purely geometric question:

Problem 8. Given compact submanifolds with boundary, say $M_{1}, \cdots, M_{k} \subset$ $\mathbb{R}^{n}$, perhaps of different dimensions, under what geometric conditions are the interiors of the submanifolds going to have a non-empty stable intersection?

Although at first this looks like a hopelessly general question, it is nevertheless possible to give a satisfactory answer that allows for some amusing global applications.

In order to guarantee that the property of non-empty intersection in Problem 8 is persistent under small deformations, and since one does not know $a$ priori, where the intersection is going to lie, it is natural to require that for all choices of points $p_{j} \in M_{j}$, the normal spaces

$$
\left[T_{p_{1}} M_{1}\right]^{\perp}, \cdots,\left[T_{p_{k}} M_{k}\right]^{\perp}
$$

are in direct sum. Starting from this natural assumption, we proved in [X2] that the intersection of all submanifolds $M_{j}$ is non-empty provided a certain estimate holds. The inequality in question involves what seems to be the three essential quantitative features of the problem:

i) The Riemannian sizes of the submanifolds (relative to the natural induced metrics).

ii) A weighed measure of the effect of the Euclidean translations.

iii) The deviation from orthogonality of all direct sums $\left[T_{p_{1}} M_{1}\right]^{\perp} \oplus \cdots \oplus$ $\left[T_{p_{k}} M_{k}\right]^{\perp}$.

For the sake of illustration, we give here the special case of the main estimate of [X2] that corresponds to two compact hypersurfaces (with boundary) contained in $\mathbb{R}^{n}$ :

(•) Let $\alpha \in\left[0, \frac{\pi}{2}\right]$ be the infimum of the angles formed by all normal lines corresponding to arbitrary points in $\operatorname{int} M_{1}$ and $\operatorname{int} M_{2}$, one point from each 
hypersurface. If $\alpha>0$ and

$$
\frac{\sqrt{1+\cos \alpha}}{1-\cos \alpha}<\sup _{q_{1} \in M_{1}, q_{2} \in M_{2}} \frac{\min \left\{d_{1}\left(q_{1}, \partial M_{1}\right), d_{2}\left(q_{2}, \partial M_{2}\right)\right\}}{\left|q_{1}-q_{2}\right|},
$$

then $\left(\operatorname{int} M_{1}\right) \cap\left(\operatorname{int} M_{2}\right) \neq \emptyset$.

The reader is invited to experiment with flat discs in $\mathbb{R}^{3}$, of various sizes and normals, in order to show that the above result is sharp.

The global consequences of the general version of the above inequality are quite pleasing, allowing one to extend the basic intersection properties of linear affine subspaces to the non-linear context:

Theorem 8 (A non-linear view of linear algebra [X2]). Let $M_{1}, \cdots, M_{k}$ be connected complete embedded non-compact smooth submanifolds, $1 \leq \operatorname{dim} M_{j}=$ $m_{j}<n, \operatorname{codim} M_{1}+\cdots+\operatorname{codim} M_{k} \leq n$. Let $\mathcal{G}_{j}: M_{j} \rightarrow G\left(n-m_{j}, n\right)$, $\mathcal{G}_{j}(p)=\left[T_{p} M_{j}\right]^{\perp}$, be the Grassmanian-valued Gauss map of $M_{j}$. Assume that for all points $E_{j}$ in the closure of $\mathcal{G}_{j}\left(M_{j}\right), 1 \leq j \leq k$, the subspaces $E_{1}, \cdots, E_{k}$ of $\mathbb{R}^{n}$ are in direct sum. Then $M_{1} \cap \cdots \cap M_{k}$ is non-empty. Moreover, this intersection reduces to a single point if codim $M_{1}+\cdots+\operatorname{codim} M_{k}=n$.

Corollary 1. If $M_{1}, M_{2}$ are complete embedded connected hypersurfaces, then

$$
\overline{\mathcal{G}_{1}\left(M_{1}\right)} \cap \overline{G_{2}\left(M_{2}\right)}=\emptyset \Rightarrow M_{1} \cap M_{2} \neq \emptyset .
$$

Corollary 2. Let $M_{1}, \cdots, M_{n}$ be complete embedded connected hypersurfaces of $\mathbb{R}^{n}$. If every hyperplane in $\mathbb{R} \mathbb{P}^{n-1} \cong G(1, n)$ intersects at most $n-1$ of the sets $\overline{\mathcal{G}_{1}\left(M_{1}\right)}, \cdots, \overline{\mathcal{G}_{n}\left(M_{n}\right)}$, then $M_{1} \cap \cdots \cap M_{n}$ consists of a single point.

As we pointed out in our remarks preceding Problem 8, there is a clear dynamical interpretation behind these results, which we now explain.

Suppose $M_{1}, \cdots, M_{k}$ are compact submanifolds with boundary, perhaps of different dimensions, but for which the sum of the codimensions does not exceed $n$. Let us assume that their intersection is empty. We fix $M_{1}$ and continuously 
translate $M_{2}, \cdots, M_{k}$ along suitable directions until they intersect $M_{1}$ at the same point, after one unit of time. The idea is to undo the motion of the submanifolds $M_{2}, \cdots, M_{k}$, in the direction of increasing times, starting say at time $t=-1$, while keeping track of the evolution of the (local) intersection set. Since we are assuming that $\bigcap_{j=1}^{k} M_{j}=\emptyset$, the backwards motion will cease to have a common intersection sometime before (or when) one unit of time has elapsed.

One wants to control the speed at which the intersection set is propagating. Of course, it is technically easier to observe the evolution of a single point (given by the flow of a vector field, say), rather than that of the entire intersection set. In order to minimize speed, we choose curves parametrized by time that move orthogonally to the intersection set. As explained, the intersection must cease to exist before (or at) time $t=0$. But this can only happen if for some submanifold $M_{j}$ the appropriate integral curve $x_{j}$ in the interior of $M_{j}$ reaches $\partial M_{j}$ before (or at) time 0 .

The speed of motion of the intersection set is controlled by the local configuration of normal spaces to the moving submanifolds. In fact, the speed increases if the normal spaces of the submanifolds tend to "rest "on each other. A somewhat similar situation occurs if one rotates a line $L_{1}$ in the plane about one of its points. The intersection $L_{1}^{\theta} \cap L_{2}$ between the line $L_{1}$ rotated by $\theta$ and a line $L_{2}$ parallel to $L_{1}$ moves faster as $\theta \rightarrow 0$. In other words, the speed of $L_{1}^{\theta} \cap L_{2}$ increases when the distortion of the configurations of their normal spaces, coming from iii) above, tends to $\infty$.

As we indicated above, one must control the distortion of the direct sum of normal spaces at the intersection set during the evolution. But since we are simply translating the submanifolds, the supremum of the distortions of the configurations of all normal spaces remains constant throughout the motion. An estimate can then be written down (the above one, in the case of two hypersurfaces), which guarantees that the intersection set in the backward 
motion will exist for more than one unit of time, a contradiction to the original assumption that the interiors of the submanifolds are disjoint.

Some form of the technique introduced in [X2] should work whenever the ambient manifold has a large enough group of isometries. In particular, one would like to know the answer to the following

Problem 9. Given compact submanifolds with boundary of either the $n$-sphere or the hyperbolic $n$-space, under what geometric conditions are the $\underline{\text { interiors }}$ of the submanifolds going to have a non-empty stable intersection?

The search for new mechanisms of global injectivity remains a lively area of investigation. The author can only hope that this short survey article will encourage others to pursue their own exploration of the subject.

\section{References}

[AG] Alexander, S.; Ghomi, M., The convex hull property and topology of hypersurfaces with non negative curvature, Adv. Math., 180 (2003),324-354.

[B1] Balreira, E. C., Foliations and global inversion, Comment. Math. Helv. (to appear)

[B2] Balreira, E. C., Imcompressibility and global inversion, Topological Methods in Nonlinear Analysis (to appear)

[BM] Bochner, S.; Martin, W., Several complex variables, Princeton Univ. Press (1948).

[Br] de Branges, L., A proof of the Bieberbach conjecture, Acta Math. 154, (1982), 137-152.

[BW] Bass, H.; Connell, E.; Wright, D., The Jacobian conjecture: reduction of the degree and formal expansion of the inverse, Bull. Amer. Math. Soc. 7, (1982), 287-330. 
[CMe] Chamberland, M.; Meisters, G. , A mountain pass to the Jacobian Conjecture, Canad. Math. Bull., 41 (1998), 442-451.

[CM1] Colding, T.; Minicozzi II, W. , The space of embedded minimal surfaces of fixed genus in a 3-manifold I; Estimates off the axis for discs, Annals of Math., 160 (2004), 27-68.

[CM2] Colding, T.; Minicozzi II, W., The space of embedded minimal surfaces of fixed genus in a 3-manifold II; Multi-valued graphs in disks, Annals of Math., 160 (2004), 69-92.

[CM3] Colding, T.; Minicozzi II, W. , The space of embedded minimal surfaces of fixed genus in a 3-manifold III; Planar domains, Annals of Math., 160 (2004), 523-572.

[CM4] Colding, T.; Minicozzi II, W. , The space of embedded minimal surfaces of fixed genus in a 3-manifold IV; Locally simply-connected, Annals of Math., 160 (2004), 573-615.

[D] Druzkowski, L., An effective approach to Keller's jacobian conjecture, Math. Ann., 264 (1983), 303-313.

[E] den Essen, V. A., Polynomial automorphisms and the jacobian conjecture, Prog. Math., Birkhauser (2000).

[Fe] Fessler, R., A proof of the two-dimensional Markus-Yamabe Conjecture and a generalization, Ann. Polon. Math., 62 (1995), 45-75.

[FX] Fontenele, F.; Xavier, F., A Riemannian Bieberbach estimate, preprint

[G] Glutsiuk, A., A complete solution of the jacobian problem for vector fields on the plane, Russian Math. Surveys, 49 (1994), 185-186.

[Gh1] Ghomi, M., Gauss map, topology, and convexity of hypersurfaces with non vanishing curvature, Topology, 41 (2002), 107-117. 
[Gh2] Ghomi, M., Strictly convex submanifolds and hypersurfaces of positive curvature, J. Differential Geom., 57 (2001), 239-271.

[GLS] Gasull, A.; Llibre, J.; Sotomayor, J., Global asymptotic stability of differential equations in the plane, Journal of Differential Equations, 91 (1991), 327-335.

[Gu] Gutierrez, C., A solution to the bidimensional global asymptotic stability conjecture, Ann. Inst. H. Poincaré Anal. Non Linéaire, 12 (1995), 627-671.

[H] Hadamard, J., Sur les transformations ponctuelles, Bull. Soc. Math. France, 34, (1906), 71-84.

[M] Miller, J., Some global inverse function theorems, Journal of Math. Anal and Appl., 100, (1984), 375-384.

[MR] Meeks, W.; Rosenberg, H., The uniqueness of the helicoid, Annals of Math., 161 (2005), 723-754.

[NTX] Nollet, S.; Taylor, L.; Xavier, F., Birationality of étale maps via surgery, Journal für die reine und angewandte Mathematik, (to appear)

[NX1] Nollet, S.; Xavier, F., Global inversion via the Palais-Smale condition Disc. Cont. Dyn. Syst. A, 8, (2002), 17-28.

[NX2] Nollet, S.; Xavier, F., Holomorphic injectivity and the Hopf map, Geom. Funct. Anal., 14, (2004), 1339-1351.

[O] Olech, C., On the global stability of an autonomous system in the plane, Contributions Diff. Equations, 1 (1963), 389-400.

[P] Plastock, R., Homeomorphisms between Banach spaces, Trans. Amer. J. Math. Soc., 200 (1974), 169-183.

[R] Rabier, P., Ehresmann fibrations and Palais-Smale conditions for morphisms of Finsler manifolds, Annals of Math., 146 (2) (1997), 647-691. 
[S] Segal, S., Nine introducions in complex-analysis, Revised edition, North Holland Mathematical Studies, Elsevier, 208, (2008).

[So] Sotomayor, J., Inversion of smooth mappings, Z. Angew. Math. Phys., 41 (1990), 306-310.

[SX] Smyth, B.; Xavier, F., Injectivity of local diffeomorphisms from nearly spectral conditions, J. Diff. Eqs., 130 (1996), 406-414.

[V] Verdier, J., Stratifications de Whitney et théoréme de Bertini-Sard, Inventiones Math., 36 (1976), 295-312.

[W] Wang, S., A jacobian criterion for separability, J. Algebra, 69 (1980), 453494.

[X1] Xavier, F., Injectivity as a transversality phenomenon in geometries of negative curvature, Illinois J. Math., 43 (1999), 256-263.

[X2] Xavier, F., Using Gauss maps to detect intersections, L'Enseignement Math., 53 (2007), 15-31.

[X3] Xavier, F., Rigidity of the Identity, Comm. Contemp. Math., 9 (2007), 691-699.

[Y] Yagzhev, A., On Keller's problem, Siberian Math. Jour., 21 (1980), 747754 .

[Z] Zampieri, G., Finding domains of invertibility for smooth functions by means of attraction basins, Journal of Differential Equations, 104 (1992), 11-19.

Department of Mathematics University of Notre Dame Notre Dame IN, 46635

E-mail: xavier.1@nd.edu 